\author{
SANDIA REPORT \\ SAND2011-0172 \\ Unlimited Release \\ January 2011
}

\title{
Molecular-Scale Measurements of Electric Fields at Electrochemical Interfaces
}

Roger L. Farrow and Carl C. Hayden

Prepared by

Sandia National Laboratories

Albuquerque, New Mexico 87185 and Livermore, California 94550

Sandia National Laboratories is a multi-program laboratory managed and operated by Sandia Corporation, a wholly owned subsidiary of Lockheed Martin Corporation, for the U.S. Department of Energy's

National Nuclear Security Administration under contract DE-AC04-94AL85000.

Approved for public release; further dissemination unlimited. 
Issued by Sandia National Laboratories, operated for the United States Department of Energy by Sandia Corporation.

NOTICE: This report was prepared as an account of work sponsored by an agency of the United States Government. Neither the United States Government, nor any agency thereof, nor any of their employees, nor any of their contractors, subcontractors, or their employees, make any warranty, express or implied, or assume any legal liability or responsibility for the accuracy, completeness, or usefulness of any information, apparatus, product, or process disclosed, or represent that its use would not infringe privately owned rights. Reference herein to any specific commercial product, process, or service by trade name, trademark, manufacturer, or otherwise, does not necessarily constitute or imply its endorsement, recommendation, or favoring by the United States Government, any agency thereof, or any of their contractors or subcontractors. The views and opinions expressed herein do not necessarily state or reflect those of the United States Government, any agency thereof, or any of their contractors.

Printed in the United States of America. This report has been reproduced directly from the best available copy.

Available to DOE and DOE contractors from

U.S. Department of Energy

Office of Scientific and Technical Information

P.O. Box 62

Oak Ridge, TN 37831

Telephone: $\quad$ (865) 576-8401

Facsimile: $\quad$ (865) 576-5728

E-Mail: reports@adonis.osti.gov

Online ordering: http://www.osti.gov/bridge

Available to the public from

U.S. Department of Commerce

National Technical Information Service

5285 Port Royal Rd.

Springfield, VA 22161

Telephone: $\quad$ (800) 553-6847

Facsimile: (703) 605-6900

E-Mail: $\quad$ orders@ntis.fedworld.gov

Online order: $\quad$ http://www.ntis.gov/help/ordermethods.asp?loc=7-4-0\#online

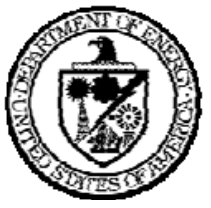


SAND2011-0172

Unlimited Release

January 2011

\title{
Molecular-Scale Measurements of Electric Fields at Electrochemical Interfaces
}

\author{
Roger L. Farrow and Carl C. Hayden \\ Combustion Chemistry Department \\ Sandia National Laboratories \\ P.O. Box 969 \\ Livermore, CA 94551
}

\begin{abstract}
Spatially resolved measurements of electric fields at electrochemical interfaces would be a critical step toward further understanding and modeling the detailed structure of electric double layers. The goal of this project was to perform proof-of-principle experiments to demonstrate the use of field-sensitive dyes for optical measurements of fields in electrochemical systems. A confocal microscope was developed that provides sensitive detection of the lifetime and high resolution spectra of excited fluorescence for dyes tethered to electrically conductive surfaces. Excited state lifetimes for the dyes were measured and found to be relatively unquenched when linked to indium tin oxide, but strongly quenched on gold surfaces. However, our fluorescence detection is sufficiently sensitive to measure spectra of submonolayer dye coatings even when the fluorescence was strongly quenched. Further work to create dye labeled interfaces on flat, uniform and durable substrates is necessary to make electric field measurements at interfaces using field sensitive dyes.
\end{abstract}




\section{CONTENTS}

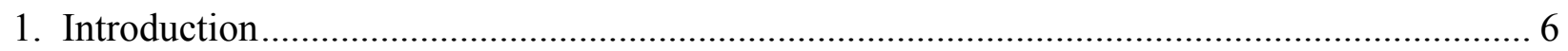

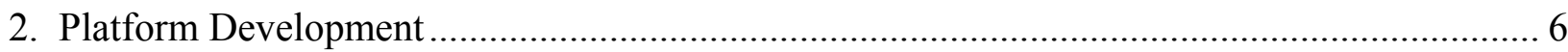

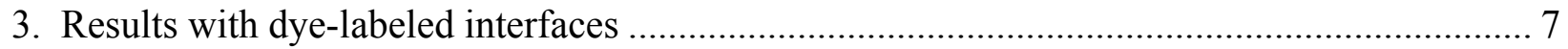

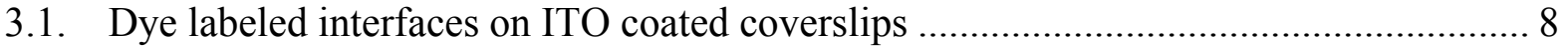

3.2. Dye labeled interfaces on gold coated coverslips ................................................... 9

3.3. Hybrid bilayers on gold coated coverslips ...................................................... 10

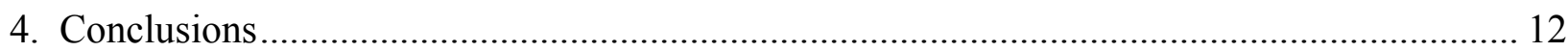

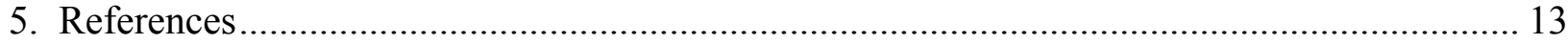

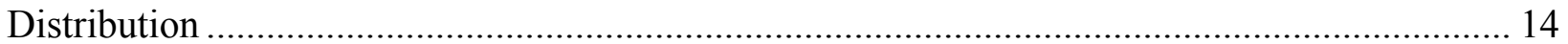




\section{NOMENCLATURE}

EDL electric double layer

CCD charge couple device

ITO indium tin oxide

NHS $\quad N$-Hydroxysuccinimide

AFM atomic force microscopy

RMS root mean square

SAM self-assembled monolayer

POPC palmitoyl oleoyl phosphatidyl choline

SUV small unilamellar vesicle 


\section{INTRODUCTION}

Electrochemical devices typically consist of an anode and cathode separated by an electrolyte. The voltage drop across the device occurs in thin layers, called electric double layers (EDLs), at the interfaces between the electrodes and electrolyte. The voltage drop might be only on the order of a volt, however, because the double layer across which the voltage drop occurs can be thinner than $10 \mathrm{~nm}$, the resulting electric field in the double layer reaches very high values. These high electric fields provide the driving forces for electrochemical processes such as charge transfer. The local fields at electrolyte/electrode interfaces are largely determined by the structure of ion distributions in the electrolyte near a charged interface. These ion distributions are influenced by the structure and chemical composition of the electrode surface along with the type of ions in the electrolyte. Thus, the EDL, which is critical to device performance, can have a complex structure that depends on many parameters. Measurements of spatially resolved electric fields in the EDL at an interface would be a critical step toward further understanding and modeling EDL structure.

One goal of this project was to perform proof-of-principle experiments to demonstrate the utility of using field-sensitive dyes for optical measurements of fields in electrochemical systems. Our approach draws on Sandia expertise in single-molecule microscopy and takes advantage of fieldsensitive (electrochromic) dyes similar to those currently coming into use for membranepotential measurements in biological systems. ${ }^{1}$ The field-sensitive dyes investigated exhibit a large change in dipole moment upon optical excitation; i.e., the dipole moment of the excited state is significantly different from that of the ground state. Because differences between upper and lower state energies vary to first order in electric field as $-\left(\mu_{\text {excited }}-\mu_{\text {ground }}\right) \cdot \mathbf{E}$, frequencies in the fluorescence spectrum shift in response to local field strength. Our fluorescence spectraland lifetime-resolved microscope provides unique capabilities to make such measurements with the sensitivity to probe individual molecules. ${ }^{2}$

A second goal of this work was the development of a platform for making in-situ optical measurements on functioning electrochemical interfaces. Optical excitation of the interface can be via either single- or two-photon absorption of light from a variety of laser sources including the fundamental or frequency-doubled pulses from a femtosecond mode-locked Ti:Sapphire laser. The laser excitation passes through a microscope objective and transparent substrate to impinge on the interface. Sensitive detection of the lifetime and spectrum of excited fluorescence are possible with the apparatus developed.

\section{PLATFORM DEVELOPMENT}

A laser excited confocal microscope was developed for this research. The apparatus was based on an inverted microscope body (Olympus IX-71) with a 60x oil immersion objective. The excitation laser was directed into the rear port of the microscope body with a long-pass dichroic mirror (Semrock) selected to reflect the laser wavelength. A totally reflecting mirror in the microscope mirror turret directed the laser through the objective into the sample. Fluorescence was collected in the backward direction through the same objective and passed through the dichroic mirror into an enclosed detection region. 
Several optical detection schemes were implemented for these experiments. The detected fluorescence was dispersed with a very high efficiency transmission diffraction grating (Wasatch Photonics) onto a megapixel Firewire CCD camera to obtain high resolution fluorescence spectra. The signal from the illuminated pixel rows was extracted from the image to yield the spectrum. This arrangement was able to readily detect fluorescent dye layers with coverage less than $1 \%$ of a monolayer. The fluorescence could also be directed into a high speed photomultiplier tube that was used with a time-correlated single photon counting system (Sensl) for fluorescence lifetime measurements. Several lasers could be used for optical excitation to provide a range of wavelengths, a diode laser at $405 \mathrm{~nm}$, a diode pumped solid state laser at 471 $\mathrm{nm}$ and the second harmonic of a femtosecond mode-locked Ti:Sapphire provided short pulses tunable from $380-470 \mathrm{~nm}$ for lifetime measurements.

A prototype optically accessible electrochemical cell was also developed for these experiments. This cell consisted of a custom machined Delrin platform that holds a microscope coverslip and a microscope slide in a sandwich geometry. The facing glass surfaces have semitransparent conductive coatings, either indium tin oxide (ITO) or gold. A gap between the surfaces is maintained by a $1 \mathrm{~mm}$ silicone spacer, which also contains the electrolyte solution. Electrical contact with the conductive interfaces is made by gold foil strips clamped between the silicone spacer and conductive coatings. Voltages were applied across the electrolyte filled cell using a lab power supply or a computer controlled analog-to-digital converter. Figure 1 shows a schematic of the cell and optical excitation scheme.

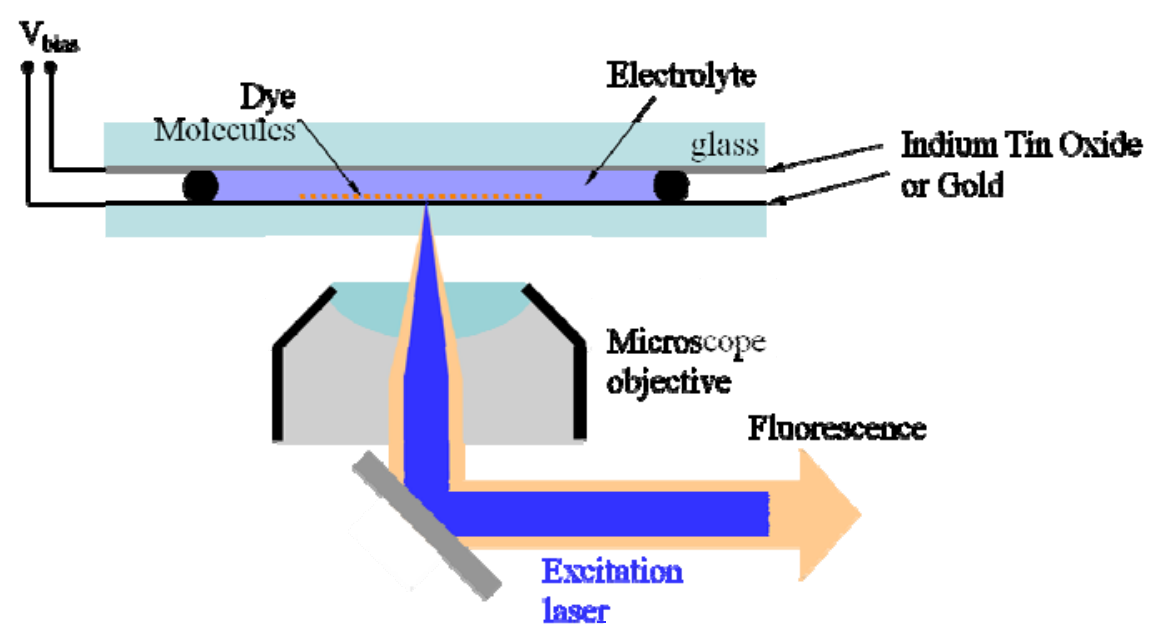

Figure 1. Schematic of the experimental cell for optical electrochemical measurements. The laser excitation path and fluorescence collection geometry are also shown.

\section{RESULTS WITH DYE-LABELED INTERFACES}

A key to our experimental approach is the development of dye labeled conductive surfaces for the optical measurements. For calibration measurements to determine the fluorescence spectral shifts as a function of electric field the dyes must be attached at a well defined height above the 
surface so the field can be predicted. We tried several different methods for fabricating suitable interfaces. The results of these approaches are described below.

\subsection{Dye labeled interfaces on ITO coated coverslips}

ITO substrates have several attractive characteristics for these experiments. ITO surfaces can be nearly completely transparent in the visible making them ideal for optical probing. The ITO coating is highly electrically conductive at low frequencies, but not at optical frequencies so dyes attached in close proximity to the surface are not expected to be strongly quenched by the coating. Also, ITO can be functionalized by silane chemistry commonly used for glass or silicon dioxide.

\section{Glass}
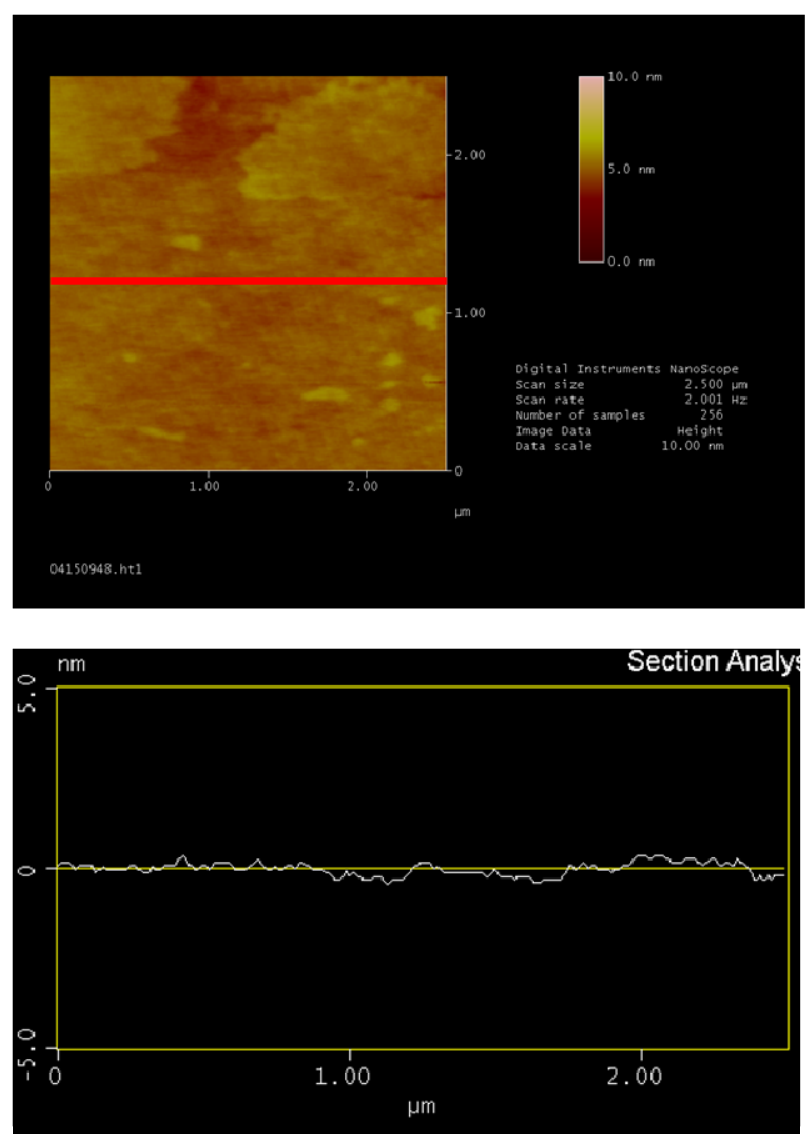
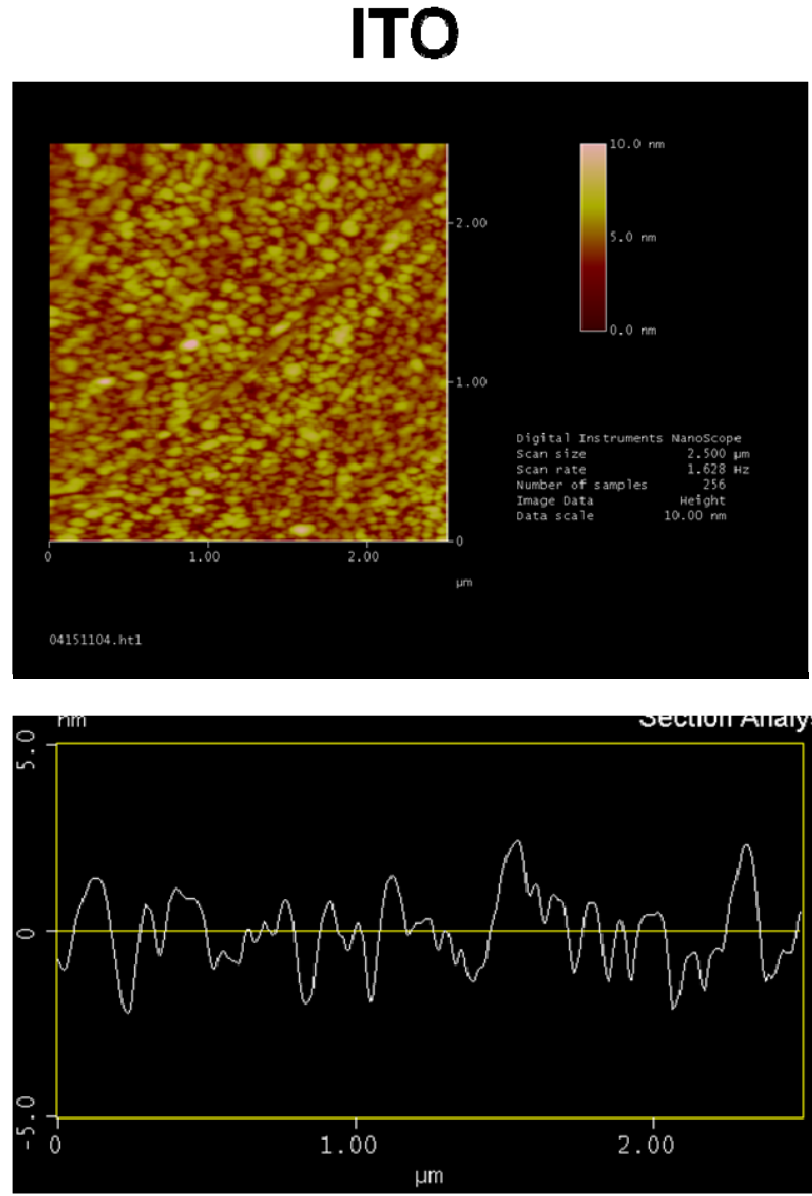

Figure 2. AFM images of ITO substrates compared to glass coverslips. The profiles across the images are shown on a -5.0 to $5.0 \mathrm{~nm}$ scale. Data from Dr. Julie A. Last, Univ. of Wisconsin-Madison

We functionalized ITO coverslips (SPI) with aminopropyltriethoxysilane (Sigma-Aldrich) in acetone solution. This treatment covalently attaches amine functional groups to the surface, which can react with NHS ester functionalized dyes commercially available for protein labeling. 
The dye carboxyrhodamine 6G NHS (Anaspec) was chosen for initial experiments. The dye was successfully attached to the surface with submonolayer coverage and was readily detected by our confocal microscope. Fluorescence lifetime measurements verified that there was minimal quenching by the ITO surface. This dye was chosen to develop the surface attachment method and was not expected to show a strong electrochomic effect. Measurements of the spectrum as a function of field applied to the interface did not show significant shifts.

An important question with the commercial ITO coatings was their surface roughness. A rough surface will place dyes at a range of heights within the EDL making calibration of the field measurements difficult or impossible. To test the surface roughness AFM measurements were made on the ITO surfaces. The surface was found to be quite structured with an RMS roughness greater than $1 \mathrm{~nm}$ and many features larger than this. Since this feature size is a significant fraction of the expected EDL thickness further development of dye labeled interfaces on ITO were not attempted. The AFM results are shown in Figure 2.

\subsection{Dye labeled interfaces on gold coated coverslips}

Gold surfaces are readily functionalized for dye attachment and have high electrical conductivity even with gold layers of $100 \AA$. These thin gold layers on coverslips are semitransparent so laser excitation and fluorescence collection are possible through the interface. A drawback of the gold surface is that the metallic conductivity extends to optical frequencies so quenching of the dye fluorescence is expected.
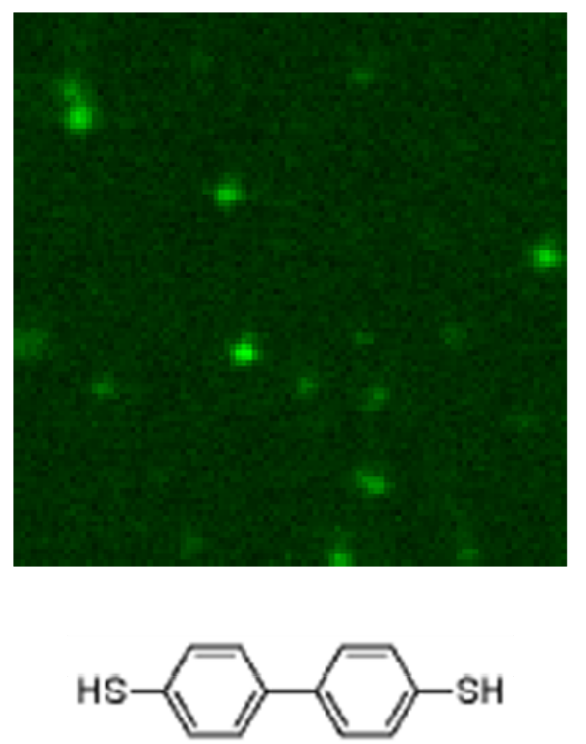

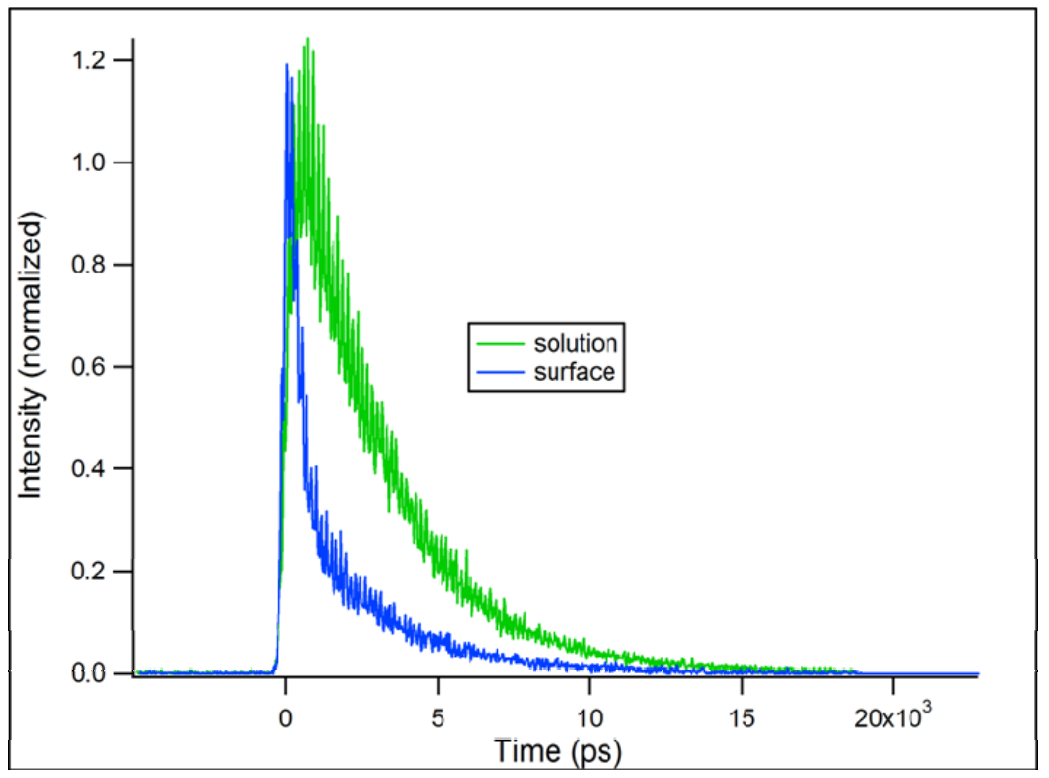

Figure 3. Confocal image of a $\sim 10 \mu \mathrm{m}$ square region of a biphenyl dithiol SAM on gold labeled with Atto 532 dye. The image shows a fairly uniform surface with some brightly labeled flaws. The plot on the right compares the fluorescence lifetime of the dye attached to the surface to that in solution. It demonstrates the strong quenching of the metal surface. 
Gold coated microscope coverslips with $100 \AA$ gold layers (Sigma-Aldrich) were first functionalized with self-assembled monolayers (SAMs) of biphenyl dithiol. This SAM was chosen because the biphenyl stacks in a predominantly upright position ${ }^{3,4}$ and forms a rigid spacer between the surface and the dye. The terminal thiol will react with commercially available dyes functionalized to react with the thiols in cysteines on proteins. The commercial dye Atto 532 maleimide (ATTO-TEC) was linked to the thiols on the SAM surface. Images of the resulting surfaces were taken using $532 \mathrm{~nm}$ excitation and the dye fluorescence lifetime measured. These results are shown in Figure 3. The reduced lifetime on the SAM surface compared to solution showed that there was substantial quenching of the dye by the proximity of the gold surface. However the dye linked to the gold was readily detected by our confocal microscope system despite the strong quenching. Thus, this is a promising approach for our optical measurements.

For field dependent measurements on the gold-SAM surface the dye acrylodan (Invitrogen) was linked to the terminal thiols. This dye is thought to have a large change in dipole moment upon excitation. To optimally excite the dye the frequency doubled output at $390 \mathrm{~nm}$ from a femtosecond mode-locked Ti:Sapphire laser operating at $780 \mathrm{~nm}$ was used. A very high fluorescence background was observed from the commercial substrates excited at this wavelength. This background fluorescence completely obscured the fluorescence from the submonolayer dye coverage. Thus field dependent measurements were not feasible.

\subsection{Hybrid bilayers on gold coated coverslips}

Hybrid bilayers were formed from an alkane thiol SAM on gold as the bottom layer with a lipid monolayer on top. Hybrid bilayers of this type have recently been developed for a number of applications including electrochemical sensors. ${ }^{5,6}$ This interface geometry has attractive features for making calibration measurements of dye response to applied electric fields. The dye is encapsulated in a nonpolar dielectric material consisting of the alkanes on the thiols and the lipid tails. This eliminates possible local ionic shielding effects that may be important in electrolyte fluids. The thickness of the dielectric region is well defined by the lengths of the alkane chains in the SAM and lipid monolayer. The applied voltage appears across this region so the field can be readily determined. Hybrid bilayers of this type have been shown to be excellent electric insulators. ${ }^{7}$

For our experiments SAMs were prepared on commercial gold coated coverslips (100 A. gold thickness) using $1 \mathrm{mM} 1$-octadecanethiol in absolute ethanol. The coverslips were left in this solution for approximately 48 hours. The SAM coated gold layers were extremely hydrophobic causing water to bead without wetting the surface. The lipid monlayer used was palmitoyl oleoyl phosphatidyl choline (POPC) to form a fluid lipid monolayer. Small unilamellar vesicles (SUVs) were prepared by sonication after hydration of dried POPC films. The SUVs in buffer solution were applied to the SAM coated gold layers. After 30 minutes the excess SUVs were rinsed away leaving the lipid monolayer that formed by self assembly on the alkanethiol SAM. The formation of hybrid bilayers was confirmed by adding $0.01 \%$ of the dye $\beta$-Bodipy $530 / 550$ C5-HPC (Invitrogen) to the SUVs during preparation. The resulting labeled bilayers were imaged with the confocal microscope. Fluorescence correlation spectroscopy was used to measure the dye diffusion within the hybrid bilayer confirming its fluid nature. Measurement of 
the fluorescence lifetime of the dye showed strong quenched indicating the dye was close to the metal surface. Results of hybrid bilayer experiments are shown in Figure 4.

To measure electric fields in the hybrid bilayer we chose the dye di-4-ANEPPS (Invitrogen). This dye is known as a reliable probe for membrane potentials in biological systems. It has several advantages for our studies. It will readily incorporate into membranes so it can be added to an existing membrane sample. The dye is nonfluorescent in aqueous solution, but brightly fluorescent in a membrane so we will only observe it if it properly incorporates into our bilayer. The excitation wavelength for di-4-ANEPPS is $\sim 470 \mathrm{~nm}$ so the excitation laser causes greatly reduced background fluorescence from the substrate compared to the shorter wavelength excitation needed for acrylodan. A disadvantage of di-4-ANEPPS is that its emission spectrum is very broad making it difficult to detect a small spectral shift. To circumvent this problem we implemented a voltage modulation lock-in detection scheme for our measurement. For this technique the voltage applied to the interface was modulated at $\sim 2 \mathrm{~Hz}$ and the acquisition of the camera frames recording the spectra was synchronized to the modulation. Spectra with the voltage off were subtracted from spectra with the voltage on to obtain the spectral shift due to the applied voltage.
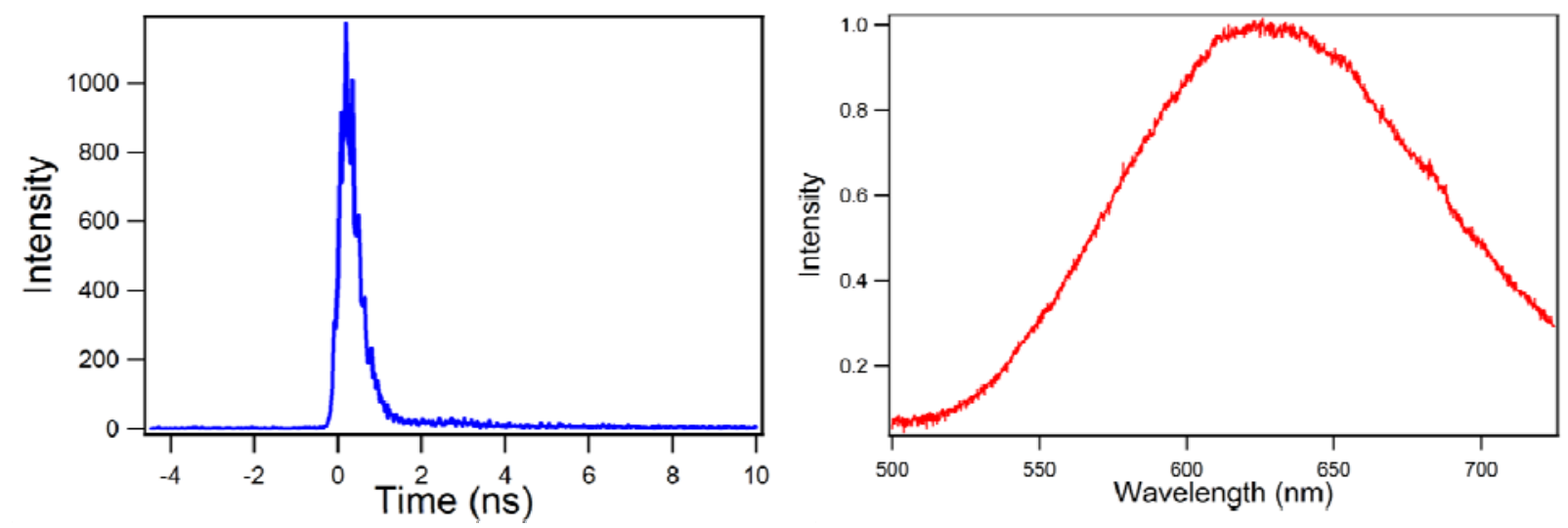

Figure 4. Results from hybrid bilayers with 1-octadecanethiol SAM and POPC monolayer on gold. The lifetime on the left was measured for the dye Bodipy 530/550 in the bilayer. The lifetime shows strong quenching by the metal surface. On the right is the spectrum of di-4-ANEPPS dye in the hybrid bilayer taken with our confocal microscope.

Experiments with di-4-ANEPPS were performed using hybrid bilayers prepared as described above but with no dye included. After the bilayer was formed a solution of $200 \mathrm{nM}$ di-4ANEPPS was added above the bilayer. After 30 minutes for the dye to incorporate into the bilayer excess dye was thoroughly rinsed off. The bilayer sample was set on the confocal microscope and excited with a $471 \mathrm{~nm}$ diode pumped solid state laser. Spectra from the labeled bilayer were easily obtained. However, the spectra were extremely sensitive to the position on the sample at the submicron level. The cause of this problem was traced to deterioration of the gold coated coverslips. The gold layer flaked off exposing the titanium adhesion layer. This process was accelerated by the procedure used to make the SAM. This project ended before we could solve this problem. 


\section{CONCLUSIONS}

We developed a very capable microscope system for optical measurements on electrochemical interfaces. The experience from this development is already being applied in new projects. The use of fluorescence dyes as probes of electric fields at interfaces is promising, but to make accurate calibration measurements of spectral shifts as a function of voltage requires careful substrate preparation. The short time scale of this project constrained us to use readily available commercial substrates. In the case of ITO on glass these were found to be too rough. The substrates with $100 \AA$ of gold on glass had high fluorescence background and insufficient durability for our experimental methods. Further development of the use of fluorescent dyes for field measurement requires improvement of interfaces used for calibration and perhaps other optical geometries that could be used with more robust (nontransparent) substrates. Thorough characterization of the functionalized interfaces and SAMs by a variety of techniques would also be important for reliable calibration measurements. 


\section{REFERENCES}

1. $\quad$ L.M. Loew, Pure and Appl. Chem. 68, 1405-1409 (1996).

2. A.K. Luong, C.C. Gradinaru, D.W. Chandler, and C.C. Hayden, J. Phys. Chem. B 109, 15691-15698 (2005).

3. A. Ulman, J.F. Kang, Y. Shnidman, S. Liao, R. Jordan, G.Y. Choi, J. Zaccaro, A.S. Myerson, M. Rafailovich, J. Sokolov, and C. Fleischer, J. Biotechnol. 74(3), 175188(2000).

4. A. Ulman, Acc. Chem. Res. 34(11), 855-63(2001).

5. A.L. Plant, Langmuir 15, 5128-5135(1999).

6. N.A. Anderson, L.J. Richter, J.C. Stephenson, and K.A. Briggman, J. Am. Chem. Soc. 129, 2094-2100( 2007).

7. S. Terrettaz, M. Mayer, and H. Vogel, Langmuir 19, 5567-5569(2003). 


\section{DISTRIBUTION}

$\begin{array}{llll}1 & \text { MS 0123 } & \text { D. Chavez, LDRD Office } & 1011 \\ 1 & \text { MS 0899 } & \text { Technical Library } & 9536 \text { (electronic copy) } \\ 1 & \text { MS 9055 } & \text { Carl Hayden } & 08353\end{array}$




\section{Sandia National Laboratories}

